\title{
A Real-World Claims Analysis of Costs and Patterns of Care in Treated Patients with Glioblastoma Multiforme in the United States
}

\author{
Andrew D. Norden, MD; Beata Korytowsky, MA; Min You, MS; T. Kim Le, MS; Homa Dastani, PhD; \\ Sarah Bobiak, PhD; and Prianka Singh, PharmD
}

\begin{abstract}
BACKGROUND: Patients with glioblastoma multiforme (GBM) have a poor prognosis and high likelihood of recurrence. Routine care for incident cases in the United States involves surgical resection, followed by radiation therapy (RT) with concurrent and adjuvant temozolomide. Real-world data reporting the treatments and health care burden associated with GBM are limited.

OBJECTIVE: To assess patterns of care, health care resource utilization (HCRU), and costs associated with treatment of GBM in the United States.

METHODS: This study is a retrospective claims database analysis. Adult patients with a GBM diagnosis (index date) between January 1, 2010, and June 30,2016 , who had undergone brain surgery within 90 days of the index date, had received temozolomide and/or RT up to 90 days after index date, and had at least 6 months of continuous enrollment before the index date, were identified. Patients were excluded if they had (a) another primary cancer within 6 months pre-index, (b) secondary brain metastases, or (c) received temozolomide and/or RT pre-index. Baseline characteristics, treatments, HCRU, and costs were reported. First-line therapy began upon first receipt of RT and/or temozolomide after index date; second-line therapy began when a new drug was added $>28$ days after initiation of first-line therapy or when there was a treatment gap $>90$ days. Treatment regimens, duration of treatment (corrected group prognosis method), HCRU, and costs were reported descriptively in the 0 - to 6 -month and 7- to 12-month periods following initiation of first-line and second-line therapy.
\end{abstract}

RESULTS: Baseline characteristics were comparable between patients receiving temozolomide and/or RT. Patients receiving RT without chemotherapy tended to be older, be retired, and have more baseline comorbidities. Of the 4,071 patients receiving first-line therapy for GBM, most $(73.0 \%)$ received temozolomide $+\mathrm{RT}$; $24.4 \%$ received $\mathrm{RT}$; and $2.5 \%$ received temozolomide monotherapy. Of those receiving first-line therapy, $1,283(31.5 \%)$ patients subsequently received second-line therapy: $39.4 \%$ received bevacizumab monotherapy; $28.9 \%$ received bevacizumab combination therapy (temozolomide, $45.2 \%$ of patients; irinotecan, $24.3 \%$; and temozolomide + lomustine, $15.4 \%) ; 15.5 \%$ received temozolomide monotherapy; and $13.7 \%$ received other systemic cancer therapies. The proportion of patients with hospitalizations increased from 2.9\% (4-6 months pre-index) to $20.8 \%$ in the 3 months before the index date (likely due to diagnostic procedures) and $28.1 \%$ in the first 6 months after index (likely due to surgery) and then decreased to $13.3 \%$ in the 7 - to 12 -month period after index. Mean total per-patient costs at 6 and 12 months were $\$ 117,325$ and $\$ 162,550$ (first line) and $\$ 126,128$ and $\$ 243,833$ (second line ). Costs in all time periods were largely driven by costs of RT/systemic cancer therapy. CONCLUSIONS: Most patients with newly diagnosed GBM received treatment according to recommendations. However, relatively few patients received second-line therapy, and the HCRU burden and costs associated with both lines of therapy were substantial. Novel therapies for GBM are required to improve treatment options and outcomes in these patients.

J Manag Care Spec Pharm. 2019;25(4):428-36

Copyright $\odot 2019$, Academy of Managed Care Pharmacy. All rights reserved.

\section{What is already known about this subject}

Glioblastoma multiforme (GBM) is an aggressive form of adult brain tumor, with a high mortality and recurrence rate The health care resource burden of patients with GBM in the United States is known to be high, but how this varies over time and line of therapy is not understood.

\section{What this study adds}

Most treated patients (73\%) receive first-line radiotherapy and concurrent temozolomide, in line with clinical guidelines.

A high proportion of patients have emergency department visits (32\%) and hospitalizations (28\%) in the 6 months after diagnosis, indicating the substantial health care resource burden associated with GBM.

Costs associated with first-line therapy for GBM are substantial, particularly in the first 6 months, and these costs increase during second-line therapy.

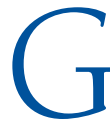
lioblastoma multiforme (GBM) is an aggressive form of adult brain tumor, with a highly unfavorable prognosis due to the near certainty of recurrence., ${ }^{1,2}$ GBM is one of the most fatal types of cancer, manifesting as a highly malignant astrocytic tumor characterized by symptoms that include cognitive impairment, neurologic deficit, and seizures, which markedly lower life expectancy and diminish patients' healthrelated quality of life., ${ }^{1,2}$

Risk factors for GBM include male sex, advancing age, white or Asian race, and the presence of a low-grade astrocytoma. ${ }^{3}$ The overall prognosis among patients with GBM remains bleak, with less than $5 \%$ of newly diagnosed patients surviving beyond 3 years. ${ }^{4,5}$ Prognosis is considerably worse for patients who experience recurrent GBM, with 6-month and 12-month overall survival estimated to be 60\%-70\% ${ }^{6-10}$ and 20\%-30\%, ${ }^{9-13}$ respectively. Despite the treatment options available to newly diagnosed patients, more than $50 \%$ of patients with GBM experience recurrence within 7 months of undergoing treatment. ${ }^{14}$ The current standard of care in the United States for newly diagnosed patients with GBM consists of surgical resection, followed by radiation therapy (RT) with concurrent and adjuvant temozolomide treatment. ${ }^{15}$ Median survival from diagnosis among patients receiving postsurgery RT-containing regimens 
has increased from 12 months to more than 14 months since the introduction of temozolomide. ${ }^{16}$

In the United States, the estimated mean annual incidence of GBM is 3.2 cases per 100,000 persons, with a median age at onset of 64 years. ${ }^{17}$ However, cost drivers and costs per line of therapy for patients with GBM are not well described in the literature. One administrative claims database analysis reported that brain cancer accounted for a mean total direct medical cost per patient per month (PPPM) of $\$ 8,478$ in the United States (inflation adjusted to 2012) ${ }^{18}$ To better understand the burden of GBM, we sought to describe treatment patterns and to quantify health care resource utilization (HCRU) and costs over time by line of therapy in a recent cohort of patients with GBM in the United States.

\section{Methods}

\section{Study Design and Setting}

A retrospective cohort study of patients diagnosed with GBM was conducted using data from Truven Health MarketScan research administrative claims databases, consisting of 148 million covered individuals in the United States, including those with private insurance as well as Medicare beneficiaries. Patients were included if they (a) were aged 18 years or older; (b) presented with a diagnosis of malignant neoplasm of the brain (International Classification of Diseases, Ninth Revision, Clinical Modification [ICD-9-CM] code 191.xx or International Classification of Diseases, Tenth Revision, Clinical Modification [ICD-10-CM] code C71.xx) between January 1, 2010, and June 30, 2016 (index date was defined as the GBM diagnosis date); (c) had undergone brain surgery within 90 days after diagnosis; (d) had received temozolomide and/or RT up to 90 days after diagnosis; and (e) had been continuously enrolled for at least 6 months before the index date. Patients were excluded from this study if they (a) had a diagnosis of another primary cancer (ICD-9/10 codes 140.xx-190.xx, 192.xx-195.xx, and 200.xx208.xx/C00.xx-C26.xx, C30.xx-C34.xx, C37.xx-C41.xx, C43.xxC58.xx, C60.xx-C70.xx, C72.xx-C76.xx, C81.xx-C85.xx, C88, and C90.xx-C97.xx); (b) had a diagnosis of secondary brain metastasis (ICD-9/10 codes 198.3/C79.3); (c) had received temozolomide and/or RT before the index date; or (d) had received any off-label or nonstandard therapy as firstline treatment. Follow-up time after the diagnosis was variable.

\section{Study Outcomes}

Demographic and clinical characteristics at baseline (i.e., prediagnosis) were obtained from the MarketScan database, including age, sex, geographic location, patient residence, health plan type, employment status, and Charlson Comorbidity Index (CCI) score (assessing comorbidities in the 6 months before diagnosis) and were reported descriptively.

The first observed line of therapy (first line) commenced upon initial receipt of temozolomide and/or RT up to 90 days after GBM diagnosis. Agents and procedures used during each line were identified by Current Procedural Terminology, 4th edition (CPT-4) codes. The regimen used in each line was considered to include all drugs given within 28 days. Line of therapy ended when a new drug was added more than 28 days after initiation of that line of therapy or if the time between each treatment exceeded 90 days, regardless of whether there was repetition of any previous therapy. Switching from one platinum-based therapy to another was not considered the end of a line of therapy.

HCRU and costs were identified by CPT-4 code and assessed by line of therapy and were reported for the 6-month pre-index period and for the 0-to 6-month and 7- to 12-month periods following initiation of first-line and second-line therapy. The 6-month pre-index period was split into 4-6 months and 0-3 months pre-index to capture the increased HCRU often seen in the 3 months before diagnosis. Total costs as well as costs for the following categories were reported: (a) medical services (outpatient, inpatient, emergency department [ED], or other medical services); (b) pharmacy services (retail or specialty pharmacy services provided under the pharmacy benefit, excluding chemotherapy); and (c) RT/systemic cancer therapy. Paid amounts (excluding negative claims from reversals) were used for calculating all health care costs incurred by patients with GBM and reported in 2016 U.S. dollars.

\section{Statistical Analyses}

Means and standard deviations (SD) were reported for continuous demographic variables. Patient counts and percentages were provided for categorical variables, including line of therapy and treatment regimen. Follow-up time from the index date and duration of treatment for each line of therapy were reported in months (median and range). Duration of therapy for each regimen class was estimated using corrected group prognosis method to adjust for significant baseline variables, and reported as median (range). All analyses were conducted in SAS software package 9.4 (SAS Institute, Cary, NC).

\section{Results}

\section{Patient Characteristics}

A total of 89,750 patients with a diagnosis code for malignant cancer of the brain were identified (Appendix, available in online article). Of these, 66,767 (74.4\%) patients did not receive surgery within 90 days after their brain cancer diagnosis, and a further 9,926 (11.1\%) patients did not receive temozolomide and/or RT and, thus, were excluded. A final count of 4,071 (4.5\%) patients met all study inclusion criteria. Mean (SD) age was 54.9 (13.3) years, with most (71.8\%) patients aged 35-64 years; $41.6 \%$ were women. Most patients resided in urban neighborhoods (83.1\%); 59.9\% were identified as being a member of a preferred provider organization insurance plan; $39.8 \%$ were active full-time employees; and $21.6 \%$ were retirees 


\section{TABLE 1 Patient Characteristics at Baseline}

\begin{tabular}{|c|c|c|c|c|c|c|c|c|}
\hline \multirow[b]{2}{*}{ Age, mean (SD), years } & \multicolumn{2}{|c|}{$\begin{array}{c}\text { All } \\
(\mathrm{N}=4,071)\end{array}$} & \multicolumn{2}{|c|}{$\begin{array}{l}\text { Radiotherapy } \\
(\mathbf{n}=995)\end{array}$} & \multicolumn{2}{|c|}{$\begin{array}{c}\text { Temozolomide } \\
(\mathrm{n}=103)\end{array}$} & \multicolumn{2}{|c|}{$\begin{array}{c}\text { Temozolomide }+ \\
\text { Radiotherapy } \\
(\mathbf{n}=2,973)\end{array}$} \\
\hline & 54.9 & (13.3) & 57.1 & (16.6) & 47.8 & $(14.4)$ & 54.4 & $(11.8)$ \\
\hline \multicolumn{9}{|l|}{ Age group, years, n (\%) } \\
\hline $18-34$ & 350 & (8.6) & 118 & (11.9) & 20 & $(19.4)$ & 212 & $(7.1)$ \\
\hline $35-64$ & 2,925 & (71.8) & 489 & $(49.1)$ & 73 & $(70.9)$ & 2,363 & $(79.5)$ \\
\hline $65-74$ & 535 & $(13.1)$ & 232 & (23.3) & 7 & $(6.8)$ & 296 & $(10.0)$ \\
\hline$\geq 75$ & 261 & $(6.4)$ & 156 & $(15.7)$ & 3 & $(2.9)$ & 102 & (3.4) \\
\hline Women, n (\%) & 1,693 & $(41.6)$ & 445 & $(44.7)$ & 39 & $(37.9)$ & 1,209 & $(40.7)$ \\
\hline \multicolumn{9}{|l|}{ Region, n (\%) } \\
\hline Northeast & 742 & (18.2) & 200 & $(20.1)$ & 18 & $(17.5)$ & 524 & $(17.6)$ \\
\hline North Central & 1,091 & $(26.8)$ & 274 & $(27.5)$ & 26 & $(25.2)$ & 791 & $(26.6)$ \\
\hline South & 1,489 & $(36.6)$ & 362 & $(36.4)$ & 35 & $(34.0)$ & 1,092 & $(36.7)$ \\
\hline West & 683 & $(16.8)$ & 146 & $(14.7)$ & 20 & (19.4) & 517 & $(17.4)$ \\
\hline Unknown & 66 & $(1.6)$ & 13 & $(1.3)$ & 4 & (3.9) & 49 & (1.6) \\
\hline \multicolumn{9}{|l|}{ Patient residence, n (\%) } \\
\hline Urban & 3,385 & $(83.1)$ & 802 & $(80.6)$ & 88 & $(85.4)$ & 2,495 & $(83.9)$ \\
\hline Rural & 623 & (15.3) & 181 & $(18.2)$ & 12 & $(11.7)$ & 430 & $(14.5)$ \\
\hline Unknown & 63 & $(1.5)$ & 12 & $(1.2)$ & 3 & $(2.9)$ & 48 & $(1.6)$ \\
\hline Medicare beneficiary, n (\%) & 746 & (18.3) & & & & & & - \\
\hline \multicolumn{9}{|l|}{ Plan type, $\mathrm{n}(\%)$} \\
\hline Health maintenance organization & 393 & $(9.7)$ & 71 & $(7.1)$ & 16 & $(15.5)$ & 306 & $(10.3)$ \\
\hline Preferred provider organization & 2,439 & $(59.9)$ & 564 & $(56.7)$ & 50 & $(48.5)$ & 1,825 & $(61.4)$ \\
\hline Other & 1,117 & $(27.4)$ & 322 & $(32.4)$ & 31 & $(30.1)$ & 764 & $(25.7)$ \\
\hline Missing & 122 & $(3.0)$ & 38 & (3.8) & 6 & $(5.8)$ & 78 & $(2.6)$ \\
\hline \multicolumn{9}{|l|}{ Employment status, n (\%) } \\
\hline Active full time & 1,620 & $(39.8)$ & 275 & $(27.6)$ & 50 & $(48.5)$ & 1,295 & $(43.6)$ \\
\hline Active part time/seasonal & 32 & $(0.8)$ & 6 & $(0.6)$ & 2 & (1.9) & 24 & $(0.8)$ \\
\hline Retiree & 881 & $(21.6)$ & 344 & $(34.6)$ & 10 & $(9.7)$ & 527 & $(17.7)$ \\
\hline Other/unknown & 1,538 & $(37.8)$ & 370 & $(37.2)$ & 41 & $(39.8)$ & 1,127 & $(37.9)$ \\
\hline CCI score, mean (SD) & 0.8 & $(1.2)$ & 0.9 & $(1.3)$ & 0.5 & $(1.1)$ & 0.8 & $(1.2)$ \\
\hline \multicolumn{9}{|l|}{ CCI score, $\mathrm{n}(\%)$} \\
\hline 0 & 2,071 & $(50.9)$ & 458 & $(46.0)$ & 67 & $(65.0)$ & 1,546 & $(52.0)$ \\
\hline $1-2$ & 1,763 & $(43.3)$ & 457 & $(45.9)$ & 32 & $(31.1)$ & 1,274 & $(42.9)$ \\
\hline $3-4$ & 134 & (3.3) & 55 & $(5.5)$ & 2 & (1.9) & 77 & $(2.6)$ \\
\hline$\geq 5$ & 103 & $(2.5)$ & 25 & $(2.5)$ & 2 & $(1.9)$ & 76 & $(2.6)$ \\
\hline \multicolumn{9}{|l|}{ CCI component, n (\%) } \\
\hline Cerebrovascular disease & 1,360 & $(33.4)$ & 347 & (34.9) & 21 & $(20.4)$ & 992 & $(33.4)$ \\
\hline Diabetes & 536 & $(13.2)$ & 175 & (17.6) & 7 & $(6.8)$ & 354 & $(11.9)$ \\
\hline Chronic pulmonary disease & 329 & $(8.1)$ & 101 & $(10.2)$ & 4 & (3.9) & 224 & $(7.5)$ \\
\hline Hemiplegia or paraplegia & 313 & $(7.7)$ & 81 & $(8.1)$ & 7 & $(6.8)$ & 225 & (7.6) \\
\hline Congestive heart failure & 121 & (3.0) & 45 & $(4.5)$ & 4 & (3.9) & 72 & (2.4) \\
\hline Metastatic solid tumor & 80 & $(2.0)$ & 18 & $(1.8)$ & 2 & (1.9) & 60 & $(2.0)$ \\
\hline Renal disease & 76 & (1.9) & 24 & $(2.4)$ & 1 & (1.0) & 51 & (1.7) \\
\hline Peripheral vascular disease & 68 & $(1.7)$ & 29 & $(2.9)$ & 1 & $(1.0)$ & 38 & $(1.3)$ \\
\hline Myocardial infarction & 56 & (1.4) & 20 & $(2.0)$ & 1 & (1.0) & 35 & (1.2) \\
\hline Liver disease & 30 & $(0.7)$ & 9 & $(0.9)$ & 2 & (1.9) & 19 & $(0.6)$ \\
\hline Rheumatic disease & 27 & $(0.7)$ & 10 & $(1.0)$ & 1 & (1.0) & 16 & $(0.5)$ \\
\hline Dementia & 19 & $(0.5)$ & 6 & $(0.6)$ & 0 & $(0.0)$ & 13 & $(0.4)$ \\
\hline Peptic ulcer disease & 12 & $(0.3)$ & 5 & $(0.5)$ & 0 & $(0.0)$ & 7 & $(0.2)$ \\
\hline AIDS/HIV & 4 & $(0.1)$ & 0 & $(0.0)$ & 0 & $(0.0)$ & 4 & $(0.1)$ \\
\hline
\end{tabular}

AIDS = acquired immune deficiency syndrome; CCI = Charlson Comorbidity Index; CVD=cardiovascular disease; HIV=human immunodeficiency virus; $S D=$ standard deviation. 


\section{FIGURE 1 Treatment Duration During the Initial 12 Months of Follow-Up According to First-Line Therapy and} Second-Line Therapy

\section{A. First-Line Therapy}

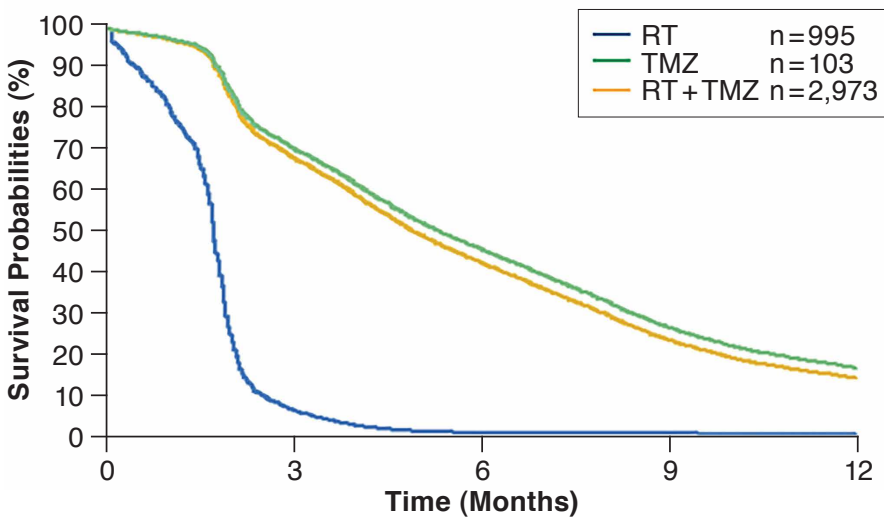

B. Second-Line Therapy

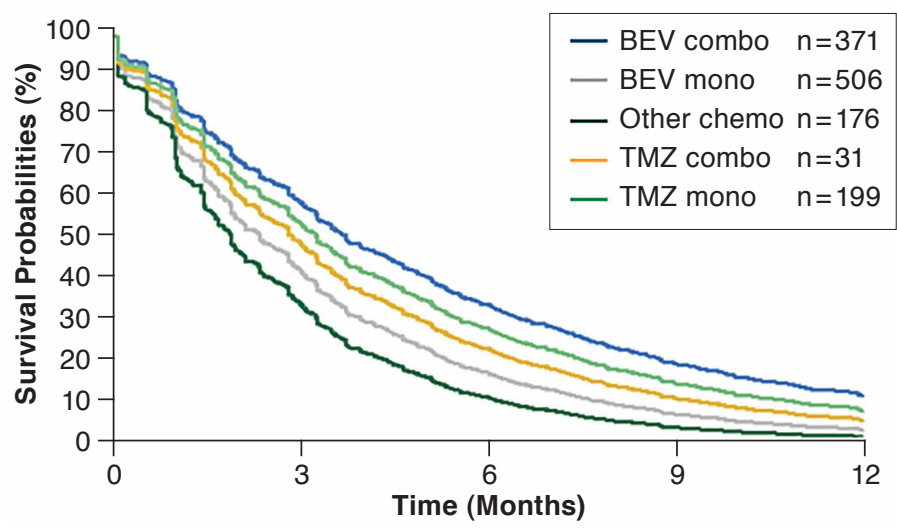

All TMZ+BEV regimens were considered as BEV combination for the purpose of this analysis. $B E V=$ bevacizumab; $R T=$ radiation therapy; $T M Z=$ temozolomide.

(Table 1). Mean baseline CCI in the 6 months before diagnosis was 0.8 , and $50.9 \%$ of patients had a baseline $\mathrm{CCI}$ of 0 in this period. The most common CCI comorbidities were diabetes (13.2\%), cerebrovascular disease (33.4\%), and chronic pulmonary disease (8.1\%). Baseline characteristics were broadly similar for patients receiving temozolomide and/or RT in first line; however, patients receiving RT only tended to be older $(39.0 \%$ of patients receiving RT only were aged 65 years or older, compared with $9.7 \%$ and $13.4 \%$ of patients receiving temozolomide only and temozolomide $+\mathrm{RT}$, respectively), generally had higher baseline mean CCI score (0.9, compared with 0.5 and 0.8 for the temozolomide only and temozolomide + RT groups, respectively), and were more likely to be retirees (34.6\% of patients, compared with $9.7 \%$ and $17.7 \%$ for the temozolomide only and temozolomide + RT groups, respectively).

\section{Patterns of Care: Treatment Regimen}

Of 4,071 patients receiving first-line therapy for GBM, the median (range) follow-up time was 11.4 (0.5-81.7) months, whereas the median (range) follow-up time from index for second-line therapy was 17.3 (3.7-81.7) months. The majority (73.0\%; median [range] duration: 5.1 [0.1-48.6] months) of firstline therapy patients received temozolomide + RT concurrently, whereas $24.4 \%$ received RT (median [range] duration: 1.7 [0.04.2] months) and 2.5\% received temozolomide monotherapy (median [range] duration: 6.4 [0.0-52.7] months). Among all patients receiving first-line therapy, 1,283 (31.5\%) received second-line therapy. The most frequently used second-line therapy was bevacizumab monotherapy (39.4\%; median [range] duration: 2.1 [0.0-42.8] months), followed by bevacizumab combination therapy (28.9\%; median [range] duration: 3.9 [0.0-64.3] months), temozolomide monotherapy (15.5\%; median [range] duration: 3.8 [0.0-42.8] months), and temozolomide combination therapy (2.4\%; median [range] duration: 4.3 [0.2-7.5] months). All other systemic therapies combined accounted for $13.7 \%$ of second-line therapies (median [range] duration: 1.4 [0.0-20.3] months). Among patients who received second-line bevacizumab in combination with another treatment, nearly half $(45.3 \%)$ received bevacizumab + temozolomide; a further $24.3 \%$ received bevacizumab + irinotecan; $15.4 \%$ received bevacizumab + temozolomide +lomustine; and $6.7 \%$ received bevacizumab + carboplatin. Several other secondline bevacizumab combination regimens were identified, but none accounted for more than $2.2 \%$ of all patients receiving second-line bevacizumab combination regimens. For patients receiving "other" systemic therapies in the second-line setting, the most commonly used regimens were lomustine $(38.6 \%$ of patients) and lomustine + procarbazine (11.4\% of patients). RT was administered concurrently with systemic therapy in $13.6 \%$ of patients receiving second-line therapy.

Analyses of duration of treatment by line of therapy are presented in Figure 1A and 1B, adjusted for significant baseline variables (age, CCI, and employment status for first line and $\mathrm{CCI}$ for second line). Almost all patients receiving first-line RT were treated for fewer than 3 months (in line with the expected treatment cycle of RT). Approximately half of patients receiving first-line temozolomide (with or without concurrent RT) were treated for fewer than 6 months, with approximately 15\% of patients still receiving therapy at 12 months. In the second-line setting, bevacizumab combination therapy and temozolomide 
TABLE 2 Health Care Resource Utilization by Line of Therapy and Time Period

\begin{tabular}{|c|c|c|c|c|c|c|c|c|}
\hline & \multicolumn{8}{|c|}{ Pre-Index Period (Time Before Diagnosis; $N=4,071$ ) } \\
\hline & \multicolumn{4}{|c|}{ 4-6 months $(n=4,071)$} & \multicolumn{4}{|c|}{$0-3$ months $(n=4,071)$} \\
\hline & $\mathbf{n}$ & $\%$ & Mean & SD & n & $\%$ & Mean & SD \\
\hline Outpatient visits & 2,681 & 65.9 & 2.63 & 3.86 & 3,569 & 87.7 & 4.85 & 4.92 \\
\hline ED visits & 207 & 5.1 & 0.07 & 0.34 & 860 & 21.1 & 0.28 & 0.66 \\
\hline Hospitalizations & 120 & 2.9 & 0.03 & 0.19 & 847 & 20.8 & 0.24 & 0.5 \\
\hline \multirow[t]{4}{*}{ Length of stay } & - & - & 5.72 & 6.53 & - & - & 3.82 & 3.58 \\
\hline & \multicolumn{8}{|c|}{ Post-Index Period, First-Line Therapy $(n=4,071)$} \\
\hline & \multicolumn{4}{|c|}{$0-6$ months $(n=1,360)$} & \multicolumn{4}{|c|}{$7-12$ months $(n=459)$} \\
\hline & $\mathrm{n}$ & $\%$ & Mean & SD & $\mathrm{n}$ & $\%$ & Mean & SD \\
\hline Outpatient visits & 1,359 & 99.9 & 34.75 & 16.96 & 459 & 100 & 19.53 & 11.76 \\
\hline ED visits & 440 & 32.4 & 0.67 & 2.3 & 91 & 19.8 & 0.3 & 0.72 \\
\hline Hospitalizations & 382 & 28.1 & 0.43 & 0.84 & 61 & 13.3 & 0.16 & 0.45 \\
\hline \multirow[t]{4}{*}{ Length of stay } & - & - & 5.57 & 5.28 & - & - & 4.24 & 3.02 \\
\hline & \multicolumn{8}{|c|}{ Post-Index Period, Second-Line Therapy $(n=1,283)$} \\
\hline & \multicolumn{4}{|c|}{ 0-6 months $(n=297)$} & \multicolumn{4}{|c|}{ 7-12 months $(\mathrm{n}=79)$} \\
\hline & $\mathrm{n}$ & $\%$ & Mean & SD & n & $\%$ & Mean & SD \\
\hline Outpatient visits & 297 & 100 & 29.67 & 17.12 & 79 & 100 & 25.25 & 13.06 \\
\hline ED visits & 100 & 33.7 & 0.58 & 1.04 & 22 & 27.8 & 0.48 & 0.95 \\
\hline Hospitalizations & 54 & 18.2 & 0.24 & 0.6 & 13 & 16.5 & 0.25 & 0.69 \\
\hline Length of stay ${ }^{a}$ & - & - & 5.36 & 6.04 & - & - & 4.85 & 3.3 \\
\hline
\end{tabular}

${ }^{a}$ Length of hospital stay was assessed in patients with at least 1 recorded hospitalization.

$E D=$ emergency department; $S D=$ standard deviation.

monotherapy were the most commonly received therapies, with approximately one third remaining on therapy at 6 months and approximately 15\% still receiving therapy at 9 months. At 12 months after second-line treatment initiation, the therapy regimen with the greatest proportion of patients still receiving treatment was bevacizumab combination therapy $(\approx 10 \%)$

\section{Patterns of Care: HCRU}

During the 4- to 6-month pre-index period, $65.9 \%$ of patients had 1 or more outpatient visits (mean [SD]: 2.6 [3.9] visits per patient). In the 3 months before the index date, the proportion of patients with outpatient visits increased to $87.7 \%$ (mean [SD]: 4.9 [4.9] visits per patient). The proportion of patients with an ED visit and hospitalization increased from 5.1\% and $2.9 \%$ during the $4-6$ months pre-index to $21.1 \%$ and $20.8 \%$ in the 3 months before the index date, respectively (Table 2).

During the first 6 months of first-line therapy and in the subsequent 7- to 12-month period, almost all patients with follow-up had outpatient visits (99.9\% and 100\%, respectively). The mean number of visits made during these periods were much greater than in the pre-index period before diagnosis, at 34.8 and 19.5 visits per patient, respectively (Table 2). The proportion of patients incurring ED visits and hospitalizations during the first 6 months after initiating first-line therapy also increased to $32.4 \%$ and $28.1 \%$, decreasing slightly in the subsequent 7 - to 12 -month period to $19.8 \%$ and $13.3 \%$, respectively, but remaining much higher than during either of the pre-index periods. Although hospitalization rates increased substantially during GBM treatment, hospitalization length of stay remained similar before diagnosis and after treatment initiation.

\section{Costs of Care}

After the first 2 months of first-line therapy, mean PPPM costs in the first line declined over the next 10 months. In the second line, PPPM costs decreased after the first month of treatment and then remained relatively stable for the next 12 months. After 4 months, more than $50 \%$ of the patients initiating either first-line or second-line treatment had been lost to follow-up. At 12 months, only $12 \%$ of patients initiating first-line therapy had follow-up; for patients initiating second-line therapy, the percentage dropped to $8 \%$

Mean total per-patient costs observed during the 4- to 6 -month pre-index period were lower $(\$ 2,927)$ than those in the 3 months before the index date $(\$ 11,021$; Figure 2). This increase was largely driven by an increase in inpatient costs from $\$ 1,418$ (48.4\% of total cost) to $\$ 7,171$ (65.1\% of total cost). Across the 6-month pre-index period, pathology/lab testing accounted for $\$ 196$ (1.4\% of total costs). Following first-line therapy initiation, mean total per-patient costs at 6 months and 12 months were $\$ 117,325$ and $\$ 162,550$, respectively, driven in large part by RT, with $72 \%$ of 12 -month costs incurred in the first 6 months. Again, the contribution of pathology/lab 
FIGURE 2 Mean Per-Patient Health Care Costs During 0-6 and 7-12 Months of First-Line Therapy and Second-Line Therapy

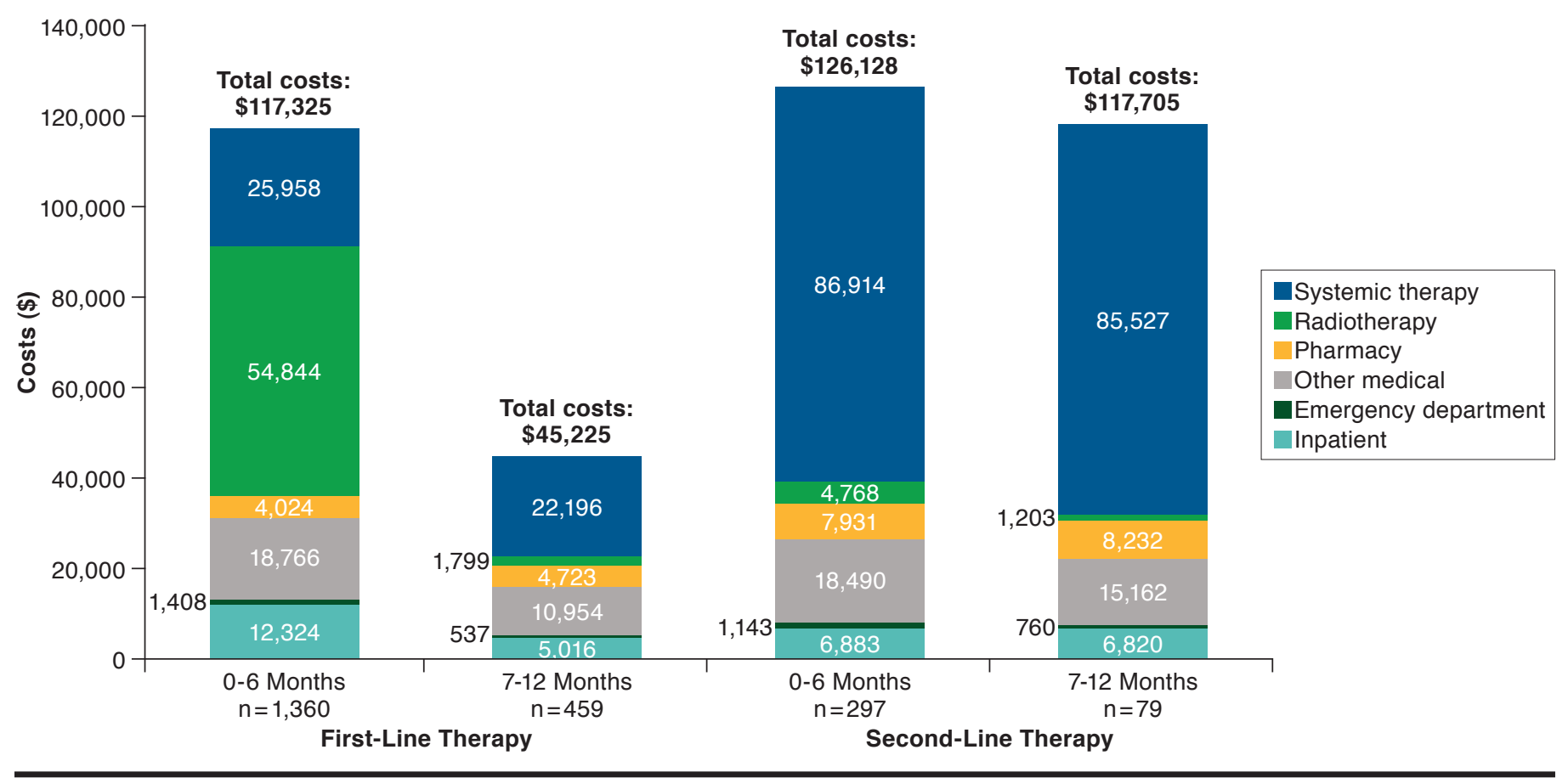

testing during this period was relatively low $(\$ 2,329 ; 1.4 \%$ of total costs). After initiating second-line therapy, mean total per-patient costs for the subsequent 6-month and 12-month periods were $\$ 126,128$ and $\$ 243,833$, respectively, mainly a consequence of systemic cancer therapy primarily attributable to bevacizumab use. Pathology/lab testing costs accounted for $\$ 2,474.47$ ( $1.0 \%$ of total costs) during the 12 months after second-line initiation.

\section{Discussion}

This large, U.S.-based, real-world study described health care treatment patterns routinely used in patients with GBM and found a substantial cost burden throughout the treatment continuum. RT and temozolomide were most often used in firstline therapy, in line with the standard of care for newly diagnosed GBM, as recommended by the National Comprehensive Cancer Network (NCCN) guidelines. ${ }^{19}$ Bevacizumab monotherapy or combination therapy was more frequently used in second-line therapy, followed by temozolomide monotherapy and chemotherapy. These trends also align with NCCN guidelines, which (in the absence of a suitable clinical trial) recommend bevacizumab, bevacizumab + chemotherapy, temozolomide, and other chemotherapies in the event of GBM recurrence, although no strict preference between these regimens is expressed. ${ }^{19}$ The high proportion of patients receiving bevacizumab monotherapy in the second-line setting may be partly due to the corticosteroid-sparing benefits seen in some patients with recurrent GBM. ${ }^{20}$

In the first-line setting, patients receiving RT alone had generally stopped receiving treatment within 3 months. This concurs with the American Society of Clinical Oncology and American Society for Radiation Oncology guidance, which recommends that RT be initiated within 3-6 weeks of diagnosis, and with a fractionation schedule typically lasting 3 or 6 weeks. ${ }^{21}$ Approximately 50\% of patients receiving temozolomide with or without concurrent RT had stopped receiving treatment by 6 months in the first-line setting. The reasons for this were beyond the scope of the current study, but most likely reflect a combination of varying clinical practice, disease progression, and poor survival outcomes for patients with GBM.,5 Among patients receiving second-line therapy, bevacizumab combination therapy and temozolomide monotherapy had the greatest proportion of patients still receiving treatment at 6 and 9 months, respectively, with bevacizumab combination having the greatest proportion of patients receiving treatment 12 months after second-line treatment initiation.

In previous analyses using Surveillance, Epidemiology, and End Results (SEER)-Medicare data, substantial proportions of patients with GBM were reported to receive no therapy. ${ }^{22,23}$ However, the earlier of these (reporting data from 1994 to 
2002) entirely preceded the influential findings of Stupp et al (2005). ${ }^{23,24}$ Although the later publication (reporting data from 1997 to 2010) captures some temozolomide use from 2005 onward, ${ }^{22}$ treatment rates remain low and may also be due to the inclusion of untreated and nonsurgical patients, further reducing the proportion receiving temozolomide $+\mathrm{RT}$, whereas our study excluded untreated and nonsurgical patients. One further publication reported observational results from the MarketScan database, as was used in the present study. ${ }^{26}$ However, that analysis lacks GBM therapy-related inclusion criteria to further target the GBM population, which has been used in the present study to mitigate inclusion of non-GBM brain tumors. A later study using retrospective chart data from patients with GBM diagnosed between 2010 and 2012 reported that $76.5 \%$ received temozolomide as their first systemic therapy after diagnosis. ${ }^{28}$ As $94.8 \%$ of these patients had received RT after diagnosis, these findings are consistent with those reported in the present study.

HCRU was high in the present study population, particularly for outpatient and ED visits and hospitalizations, with patients often using these services multiple times in the 12 months following GBM diagnosis. Additionally, increased HCRU was observed during the pre-index period, with inpatient and outpatient visits rising sharply in the 3 months immediately before diagnosis, presumably because of the appearance of symptoms and consequent diagnostic procedures. The observed increase in HCRU in the first 6 months after index is likely to be because of surgical procedures administered after diagnosis. By line of therapy, the primary cost drivers for patients with GBM were RT during first-line treatment and systemic cancer therapy attributed to bevacizumab use during second-line treatment; medical costs inclusive of hospitalizations were high, irrespective of line of therapy.

In first-line and second-line settings, PPPM costs were highest in the first 2 months after initiating therapy. The 12-month costs in the first-line setting were unevenly distributed, with the majority of costs incurred in the first 6 months, whereas the costs of second-line therapy were spread more uniformly across the first 12 months of second-line therapy. The perpatient economic burden of treating GBM is in excess of that estimated for treatment of muscle invasive bladder cancer, ${ }^{29}$ metastatic head and neck cancer, ${ }^{30}$ and chronic lymphocytic leukemia. ${ }^{31}$ From a payer perspective, it should be noted that the number of patients with follow-up (and thus contributing to aggregate population costs) in both first-line and second-line settings declined rapidly over time, with approximately 90\% of patients lost to follow-up by 12 months, most likely owing to poor survival. The progressive decline of patients with follow-up and high costs of care during the 12-month followup period illustrates the substantial cost and mortality burden of GBM and the urgent need for new treatment modalities to improve survival prospects in this population.
A key strength of this retrospective study is that it enriches our understanding of real-world clinical treatment patterns, HCRU, and associated costs in the commercially insured population compared with the controlled conditions of a clinical trial. To our knowledge, this is the largest real-world analysis of treatment patterns, costs, and HCRU in U.S. patients with GBM and the first to assess changes in costs over time.

\section{Limitations}

Several limitations regarding this analysis should be noted. First, information derived from administrative claims data is collected for the purpose of reimbursement and not for analysis. Therefore, the results from this study are dependent upon the accuracy and completeness of the data recorded. In addition, the reason for loss to follow-up is not known.

Second, identification of GBM diagnosis was limited by the broad ICD-9-CM code employed for all malignant brain cancers. Although additional inclusion and exclusion criteria were used to better identify the target population, some patients may have been misclassified as GBM or may have been omitted from the analysis despite having GBM. Given these limitations, GBM was further identified through the use of drug proxies, and as a result, this study cannot provide insight regarding outcomes of the untreated GBM population. This approach is consistent with other SEER-Medicare analyses assessing similar target populations. ${ }^{26,27}$

Third, it is possible that patients receiving therapy outside the 3-month window used in this study would have been excluded from analysis. However, such untreated patients are likely to have advanced disease and extremely dismal prognoses, ${ }^{25}$ and a similar 3-month identification window has been used in other SEER-Medicare reports assessing this target population. . $^{2,26,27}$

A fourth limitation concerns the absence of survival data in claims analyses, with consequent lack of clarity on the reason for loss to follow-up. Although it may be possible that patients may have changed employers or discontinued health care coverage, given the low survival reported for GBM from other studies, mortality was the most likely reason for the large proportion of patients lost to follow-up.

Finally, given that the present study describes and quantifies real-world practice patterns for only those GBM patients with employer-sponsored health care coverage and/or Medicare supplemental coverage, the results may not be generalizable to the overall GBM population in the United States.

\section{Conclusions}

This large real-world study of patients diagnosed with GBM underlines extensive HCRU including outpatient visits, ED visits, hospitalizations, and associated costs, especially in the 6 months after initiating first-line therapy. Despite the substantial HCRU and cost burden of this disease and the sharp decline 
in patients with follow-up, efficacious therapies for treating GBM remain limited. Only $31.5 \%$ of patients with GBM receive second-line therapy, and among those who receive first-line therapy, treatment duration remains short, indicating potential disease progression and mortality. Lack of availability of efficacious therapies in this clinical space constitutes an area of high unmet need and is a prime target for accelerated approval. A recent retrospective cohort study found that rapid access to immuno-oncology agents has significantly improved outcomes relative to patients who received standard-of-care treatment. ${ }^{32}$ Novel therapies are needed to improve patient outcomes and offset the substantial burden of disease among patients with GBM.

\section{Authors}

ANDREW D. NORDEN, MD, Dana-Farber/Brigham and Women's Cancer Center, Boston, Massachusetts. BEATA KORYTOWSKY, MA; MIN YOU, MS; T. KIM LE, MS; HOMA DASTANI, PhD; SARAH BOBIAK, PhD; and PRIANKA SINGH, PharmD, Bristol-Myers Squibb, Princeton, New Jersey.

AUTHOR CORRESPONDENCE: Prianka Singh, PharmD, Bristol-Myers Squibb, 3401 Princeton Pike, Lawrence, NJ 08648. Tel.: 609.302.3721; E-mail: Prianka.singh@bms.com.

\section{DISCLOSURES}

This study was funded by Bristol-Myers Squibb (Princeton Pike, NJ). Neither honoraria nor payments were provided for authorship. Norden received consultancy fees relating to this study from Bristol-Myers Squibb. Dastani, Korytowsky, Le, Singh, and You are employees of Bristol-Myers Squibb. Dastani and Korytowsky are shareholders of Bristol-Myers Squibb. Bobiak was an employee of Bristol-Myers Squibb at the time of this study.

Preliminary data from this study were previously presented at the International Society for Pharmacoeconomics and Outcomes Research 22nd Annual International Meeting in Boston, MA, May 20-24, 2017.

\section{ACKNOWLEDGMENTS}

Editorial assistance was provided by Karen E. Smoyer, PhD; Martin Bell, PhD; and Bríain Ó Hartaigh, PhD, of Evidence Scientific Solutions (Philadelphia, $\mathrm{PA}$ ), and funded by Bristol-Myers Squibb.

\section{REFERENCES}

1. Mischel PS, Cloughesy TF. Targeted molecular therapy of GBM. Brain Pathol. 2003;13(1):52-61.

2. Schwartzbaum JA, Fisher JL, Aldape KD, Wrensch M. Epidemiology and molecular pathology of glioma. Nat Clin Pract Neurol. 2006;2(9):494-503.

3. Mount Sinai. Brain tumor-primary-adults. 2019. Available at: http:// www.mountsinai.org/patient-care/health-library/diseases-and-conditions/ glioblastoma-multiforme. Accessed February 15, 2019.
4. Affronti ML, Heery CR, Herndon JE, 2nd, et al. Overall survival of newly diagnosed glioblastoma patients receiving carmustine wafers followed by radiation and concurrent temozolomide plus rotational multiagent chemotherapy. Cancer. 2009;115(15):3501-11.

5. Polivka J Jr, Polivka J, Rohan V, Topolcan O, Ferda J. New molecularly targeted therapies for glioblastoma multiforme. Anticancer Res. 2012;32(7):2935-46.

6. Balmaceda C, Peereboom D, Pannullo S, et al. Multi-institutional phase II study of temozolomide administered twice daily in the treatment of recurrent high-grade gliomas. Cancer. 2008;112(5):1139-46.

7. Gil MJ, de las Peñas R, Reynés G, et al. Bevacizumab plus irinotecan in recurrent malignant glioma shows high overall survival in a multicenter retrospective pooled series of the Spanish Neuro-Oncology Research Group (GEINO). Anticancer Drugs. 2012;23(6):659-65.

8. Kreisl TN, Smith P, Sul J, et al. Continuous daily sunitinib for recurrent glioblastoma. J Neurooncol. 2013;111(1):41-48.

9. Silvani A, Lamperti E, Gaviani P, et al. Salvage chemotherapy with procarbazine and fotemustine combination in the treatment of temozolomide treated recurrent glioblastoma patients. J Neurooncol. 2008;87(2):143-51.

10. van den Bent MJ, Brandes AA, Rampling R, et al. Randomized phase II trial of erlotinib versus temozolomide or carmustine in recurrent glioblastoma: EORTC brain tumor group study 26034. J Clin Oncol. 2009;27(8):1268-74.

11. Stupp R, Wong ET, Kanner AA, et al. NovoTTF-100A versus physician's choice chemotherapy in recurrent glioblastoma: a randomised phase III trial of a novel treatment modality. Eur J Cancer. 2012;48(14):2192-202.

12. Taal W, Oosterkamp HM, Walenkamp AM, et al. Single-agent bevacizumab or lomustine versus a combination of bevacizumab plus lomustine in patients with recurrent glioblastoma (BELOB trial): a randomised controlled phase 2 trial. Lancet Oncol. 2014;15(9):943-53.

13. Wick A, Felsberg J, Steinbach JP, et al. Efficacy and tolerability of temozolomide in an alternating weekly regimen in patients with recurrent glioma. J Clin Oncol. 2007;25(22):3357-61.

14. Iacob G, Dinca EB. Current data and strategy in glioblastoma multiforme. J Med Life. 2009;2(4):386-93.

15. Cabrera AR, Kirkpatrick JP, Fiveash JB, et al. Radiation therapy for glioblastoma: executive summary of an American Society for Radiation Oncology Evidence-Based Clinical Practice Guideline. Pract Radiat Oncol. 2016;6(4):217-25.

16. Johnson DR, O'Neill BP. Glioblastoma survival in the United States before and during the temozolomide era. J Neurooncol. 2012;107(2):359-64

17. Thakkar JP, Dolecek TA, Horbinski C, et al. Epidemiologic and molecular prognostic review of glioblastoma. Cancer Epidemiol Biomarkers Prev. 2014;23(10):1985-96

18. Chang S, Long SR, Kutikova L, et al. Estimating the cost of cancer: results on the basis of claims data analyses for cancer patients diagnosed with seven types of cancer during 1999 to 2000. J Clin Oncol. 2004;22 (17):3524-30.

19. National Comprehensive Cancer Network. NCCN Clinical Practice Guidelines in Oncology (NCCN Guidelines): central nervous system cancers. Version.2.2018. November 26, 2018. Available at: https://www.nccn.org/professionals/physician_gls/pdf/cns.pdf. Accessed February 15, 2019.

20. Vredenburgh JJ, Cloughesy T, Samant M, et al. Corticosteroid use in patients with glioblastoma at first or second relapse treated with bevacizumab in the BRAIN study. Oncologist. 2010;15(12):1329-34.

21. Sulman EP, Ismaila N, Armstrong TS, et al. Radiation therapy for glioblastoma: American Society of Clinical Oncology Clinical Practice Guideline endorsement of the American Society for Radiation Oncology Guideline. J Clin Oncol. 2017;35(3):361-69.

22. Chang SM, Parney IF, Huang W, et al. Patterns of care for adults with newly diagnosed malignant glioma. JAMA. 2005;293(5):557-64. 
23. Iwamoto FM, Reiner AS, Panageas KS, Elkin EB, Abrey LE. Patterns of care in elderly glioblastoma patients. Ann Neurol. 2008;64(6):628-34.

24. Stupp R, Mason WP, van den Bent MJ, et al; European Organisation for Research and Treatment of Cancer Brain Tumor and Radiotherapy Groups and the National Cancer Institute of Canada Clinical Trials Group. Radiotherapy plus concomitant and adjuvant temozolomide for glioblastoma. N Engl J Med. 2005;352(10):987-96.

25. Pretanvil JA, Salinas IQ, Piccioni DE. Glioblastoma in the elderly: treatment patterns and survival. CNS Oncol. 2017;6(1):19-28.

26. Burton E, Ugiliweneza B, Woo S, Skirboll S, Boaky M. A Surveillance, Epidemiology and End Results-Medicare data analysis of elderly patients with glioblastoma multiforme: treatment patterns, outcomes and cost. Mol Clin Oncol. 2015;3(5):971-78.

27. Ray S, Bonafede MM, Mohile NA. Treatment patterns, survival, and healthcare costs of patients with malignant gliomas in a large US commercially insured population. Am Health Drug Benefits. 2014;7(3):140-49.
28. Girvan AC, Carter GC, Li L, et al. Glioblastoma treatment patterns, survival, and healthcare resource use in real-world clinical practice in the USA. Drugs Context. 2015;4:pii: 212274.

29. Cooksley CD, Avritscher EB, Grossman HB, et al. Clinical model of cost of bladder cancer in the elderly. Urology. 2008;71(3):519-25.

30. Kim Le T, Winfree KB, Yang H, et al. Treatment patterns and economic burden of metastatic and recurrent locally-advanced head and neck cancer patients. J Med Econ. 2012;15(4):786-95.

31. Frey S, Blankart CR, Stargardt T. Economic burden and quality-of-life effects of chronic lymphocytic leukemia: a systematic review of the literature. Pharmacoeconomics. 2016;34(5):479-98.

32. O'Connor JM, Fessele KL, Steiner J, et al. Speed of adoption of immune checkpoint inhibitors of programmed cell death 1 protein and comparison of patient ages in cinical practice vs pivotal clinical trials. JAMA Oncol. 2018;4(8):el80798. 


\section{APPENDIX Cohort Selection Process and Attrition}

Diagnosis of malignant cancer of the brain $\mathrm{N}=89,750(100 \%)$

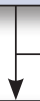

Surgery within 90 days of diagnosis $n=22,983(26 \%)$

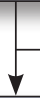

Received TMZ, RT, or both $n=13,057(15 \%)$

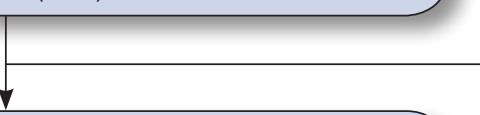

Aged $\geq 18$ years

$n=12,539(14 \%)$

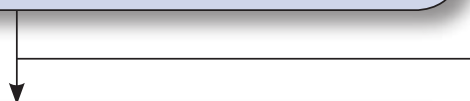

$\geq 6$ months of continuous enrollment $n=8,417(9 \%)$

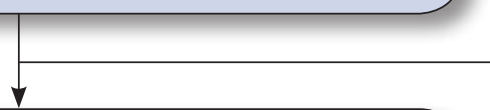

Single primary diagnosis $\mathrm{n}=4,751(5 \%)$

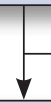

No secondary brain metastases before index date $n=4,383(5 \%)$

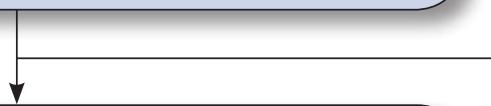

No TMZ before diagnosis $n=4,383(5 \%)$

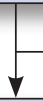

No off-label or nonstandard therapy as first line $n=4,073(5 \%)$

\section{Patients with TMZ or RT as first-line treatment $\mathrm{N}=4,071(5 \%)$}

No surgery within 90 days of diagnosis $\mathrm{n}=66,767$

Did not receive $\mathrm{TMZ}, \mathrm{RT}$, or both $n=9,926$

Aged $<18$ years

$n=518$

$<6$ months of continuous enrollment $n=4,122$

Secondary neoplasm $n=3,666$

Secondary brain metastases before index date $n=368$

TMZ before diagnosis

$n=3$

Off-label or nonstandard therapy as first line $\mathrm{n}=307$

Received TMZ or RT in lines other than first $\mathrm{n}=2$ 